\title{
Structural and Dielectric Characterization of Nickel-Cobalt Oxide Nanocomposite
}

\author{
Dedhila Devadatha* and Raveendran $\mathbf{R}$
}

Nanoscience Research Laboratory, Department of Physics, Sree Narayana College, Kollam, Kerala

\begin{abstract}
In the present work nickel-cobalt oxide nanocomposite were prepared using well known co-precipitation method. For comparison nano nickel oxide and nano cobalt oxide was also prepared. The samples were annealed at $500^{\circ} \mathrm{C}$ and characterized using SEM, TG, FTIR, XRD and UV. The dielectric studies at various frequencies of nickel-cobalt oxide nanocomposite were also done. The results showed that the capacitance of the as prepared and the annealed samples varied from milli farad to pico farad range. From this study it can be concluded that the present material by proper tuning can be used as a supercapacitor.
\end{abstract}

Keywords: Nanostructure; Nanocomposite; Chemical coprecipitation; Capping agent

\section{Introduction}

During the last few years, synthesis of nano structured oxide materials had attracted considerable attention [1]. The metal oxides are extremely important technological materials for use in electronic and photonic devices, in chemical industries and in medical fields [2]. An expanding trend for the nanomaterials is the fabrication of composite structures and devices with materials capable of enhancing the properties of the composite material. This can be done either by utilizing the size advantage through templating on the nanomaterials or by enhancing the properties to drive new synergetic properties of the two combined materials. It is also found that the magnetic nano particles provide unprecedented levels of new functionality for nano medicine. The magnetic elements like iron, nickel, cobalt and their chemical compounds are commonly present in magnetic nano particles [3]. After surface modification these materials provide both biocompatibility and functionality.

The transition metal oxides have various manufacturing possibilities based on their different properties such as anodic electrochromasim, excellent durability, electrochemical stability and large spin optical density. The conventional capacitors such as electrostatic and electrolytic capacitors cannot fulfil the needs of today's computer world as their utility is limited to certain specific applications. The next generation electrochemical double layer capacitor has been developed using transition metal oxide as one of the electrodes along with carbon. This capacitor is expected to deliver both the desirable power and energy densities [4].

Different methods have been reported for the synthesis of these nanoparticles and nanocomposite such as evaporation, magnetron sputtering, and sol-gel. In the present work a nanocomposite of nickelcobalt oxide was prepared and their modification in various known properties has been studied. Among the various methods, here we have used the famous co-precipitation method for the synthesis of nickelcobalt oxide nanocomposite.

\section{Materials and Methods}

AR grade chemicals obtained from Merck were used for the preparation of the nano particles of nickel oxide, cobalt oxide and their composites. Both the individual nano oxides and the composites were prepared by the co- precipitation method. Nickel nitrate hexahydrate, cobalt nitrate hexahydrate, and sodium hydroxide were used as starting materials.Citric acid was used as stabilizer. Aqueous solutions of 0.1 $\mathrm{M}$ nickel nitrate, $0.1 \mathrm{M}$ of cobalt nitrate and $1 \mathrm{M}$ of sodium hydroxide were slowly mixed drop wise into a beaker containing aqueous solution of citric acid and stirred well using a magnetic stirrer for two hours. The nickel-cobalt hydroxide precipitate formed was washed several times in distilled water to free it from ions and other impurities. The wet precipitate obtained was dried at room temperature and thoroughly grounded using an agate motor to obtain the nickelcobalt hydroxide precursor in the form of fine powder. The powder so obtained was annealed at $500^{\circ} \mathrm{C}$ for 3 hours in muffle furnace to obtain nanocomposite of nickel-cobalt oxide. For comparing the properties of nanocomposite with its counterpart's individual nano oxides of nickel oxide and cobalt oxide were also prepared using the same procedure.

The surface morphology of the powdered sample was obtained by Scanning Electron Microscope (SEM) [JEOL/EO JSM-6390]. FTIR studies were made using Perkin-Elmer FTIR Spectro Photo Meter in the wave number range $400 \mathrm{~cm}^{-1}$ and $4000 \mathrm{~cm}^{-1}$ by $\mathrm{KBr}$ disc method. XRD study was carried out using XPERT-PRO model powder diffractometer (PAN analytical, Netherlands) employing $\mathrm{Cu}-\mathrm{K}_{\alpha}$ radiation $(\lambda=1.54060$ $\mathrm{A}^{\circ}$ ) operating at $40 \mathrm{kV}, 30 \mathrm{~mA}$. TG analysis was carried out by PerkinElmer, diamond TGA/DTA apparatus. UV study was carried out by Schimadzu 160 A Spectrophotometer and PL study was carried out by Horiba Jobin Yvon Flurolog 3 modular Spectroflurometer. AC conductivity studies were done using Gain Phase Impedance Analyzer, MODEL HP, 4294 A, 40 Hz-110 MHz.

\section{Results and Discussions}

The samples were annealed at different temperatures and characterized using SEM, TG, FTIR, XRD, UV and PL. The dielectric

*Corresponding author: Dedhila Devadatha, Nanoscience Research Laboratory, Department of Physics, Sree Narayana College, Kollam, Kerala, E-mail: dedhila@yahoo.com

Received November 22, 2012; Accepted December 29, 2012; Published January 06, 2013

Citation: Devadatha D, Raveendran R (2013) Structural and Dielectric Characterization of Nickel-Cobalt Oxide Nanocomposite. J Material Sci Eng S11:003. doi:10.4172/2169-0022.S11-003

Copyright: (c) 2013 Devadatha D, et al. This is an open-access article distributed under the terms of the Creative Commons Attribution License, which permits unrestricted use, distribution, and reproduction in any medium, provided the original author and source are credited. 
studies at various frequencies of nickel-cobalt oxide nanocomposite were also done.

SEM analysis of nickel oxide, cobalt oxide and the nanocomposite are shown in figure 1. SEM image of nickel oxide showed aggregates of smooth solids of different size and shape. Cobalt oxide also showed an aggregation of non uniform solids. The morphology of nickel-cobalt oxide is shown in figure 1c. From SEM image it is clear that particles had undergone agglomeration.

Figure 2 depicts the typical TGA curves of the nickel oxide, cobalt oxide and nickel-cobalt oxide nanocomposite. For Nickel oxide [5] the TGA curve has two sharp weight losses. The former corresponds to the evaporation of the adsorbed and intercalated water molecules and the latter is associated with the loss of water produced by dehydroxylation of the hydroxide layers. In the case of cobalt oxide [6], the first peak indicates the departure of physically sorbed water molecules; the next peaks correspond to the loss of water of constitution and decomposition. The final weight loss is the decomposition of $\mathrm{Co}_{3} \mathrm{O}_{4}$ into $\mathrm{CoO}$ corresponds to the equation given below.

$$
2 \mathrm{Co}_{3} \mathrm{O}_{4} \rightarrow 6 \mathrm{CoO}+\mathrm{O}_{2}
$$

The TG curve for the nanocomposite shows that the material exists as a single unit at higher temperature. The weight loss due to decomposition of $\mathrm{Co}_{3} \mathrm{O}_{4}$ into $\mathrm{CoO}$ is not seen.

Figure 3 depicts the FTIR spectrum of the nickel oxide, cobalt oxide and nickel-cobalt oxide nanocomposite. The prominent bands observed in the spectrum for nickel oxide includes: $3421 \mathrm{~cm}^{-1}$ due to hydrogen-bonded hydroxyl groups, $1631 \mathrm{~cm}^{-1}$ due to bending vibration of water molecules. The weak bands at $421 \mathrm{~cm}^{-1}$ were attributed to metal oxide vibrations. In the case of cobalt oxide, the corresponding peaks were observed at peak positions $3432 \mathrm{~cm}^{-1}, 1634 \mathrm{~cm}^{-1}$ and 863 $\mathrm{cm}^{-1}$ to $430 \mathrm{~cm}^{-1}$ respectively. For the nanocomposite the corresponding peak positions were at $3426 \mathrm{~cm}^{-1}, 1627 \mathrm{~cm}^{-1}$ and $865 \mathrm{~cm}^{-1}$ to $565 \mathrm{~cm}^{-1}$.

Figure 4 shows the XRD spectrum of all the three samples. The phase purity of all the three samples was established by comparison of the X-ray diffraction pattern with JCPDS International data value. Nickel oxide was in matching with JCPDS Card no \#78-0423, Cobalt oxide with \#74-2120 and the nanocomposite was matched with \#731704. From the JCPDS Card both nickel oxide and the nanocomposite had cubic system with FCC lattice. Cobalt oxide also had cubic system. The lattice constants for all the three samples were calculated and were in agreement with JCPDS Card values. From JCPDS for nickel oxide $\mathrm{a}=4.179$, for cobalt oxide $\mathrm{a}=8.084$ and for nickel-cobalt oxide, $\mathrm{a}=9.387$.

From the XRD analyses the particle size were calculated using Scherrer equation (1).

$$
\mathrm{D}=\mathrm{k} \lambda /\left(\beta_{\mathrm{hkl}}\right) \text { measured }{ }^{\star} \cos \Theta_{\mathrm{hkl}}
$$

Here, $\mathrm{D}$ is the average crystallite size normal to the reflecting planes, $\mathrm{k}-$ is the shape factor which lies between 0.95 and 1.15 depending on the shape of the grains ( $\mathrm{k}=1$ for spherical crystallites), $\lambda$-is the wavelength of $\mathrm{x}$-ray used and $\left(\beta_{\mathrm{hkl}}\right)_{\text {measured }}$ is the FWHM of the diffraction line in radians and $\theta_{\mathrm{hkl}}$ is the Bragg angle corresponding to the diffraction line arising from the planes designated by Miller indices (hkl). From the crystallite size calculation the average size of nickel

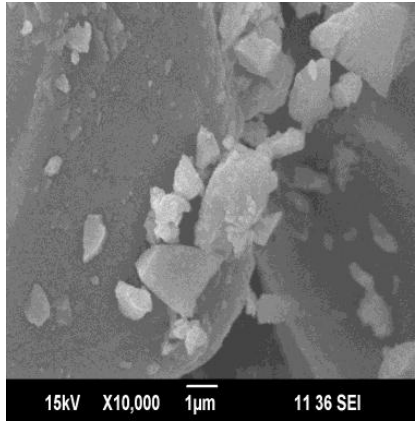

a

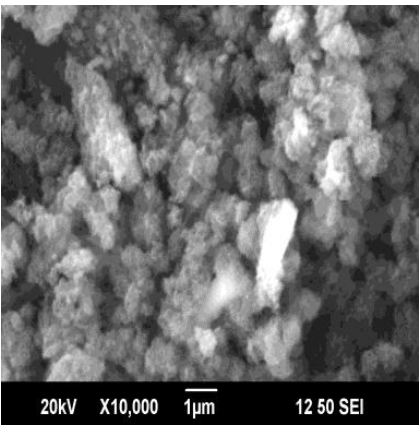

$\mathrm{b}$

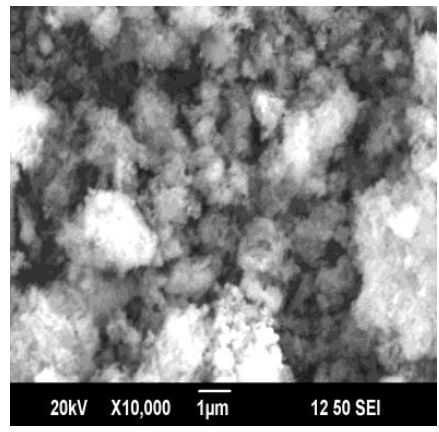

$\mathrm{c}$

Figure 1: SEM image of nickel oxide (a), cobalt oxide (b) and nickel-cobalt oxide (c)


Figure 2: TG Curve of nickel oxide (a), cobalt oxide (b) and nickel-cobalt oxide (c). 




a

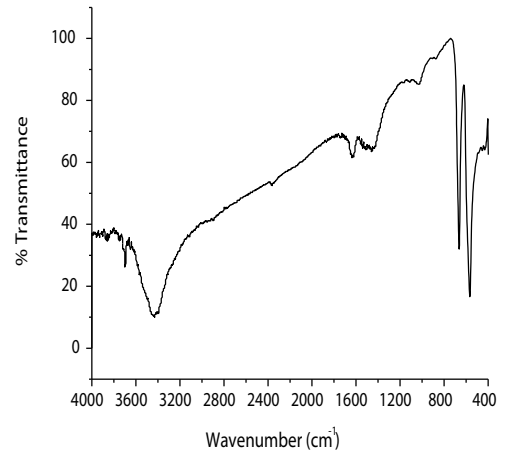

b

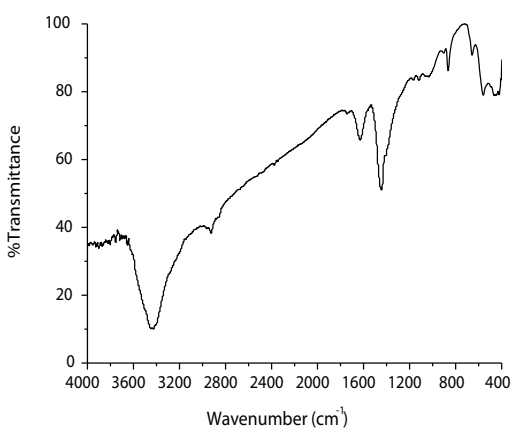

C

Figure 3: FTIR spectrum of nickel oxide (a), cobalt oxide (b) and nickel-cobalt oxide (c).

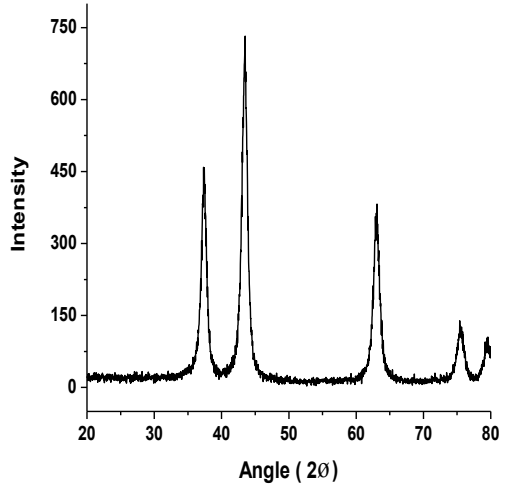

a

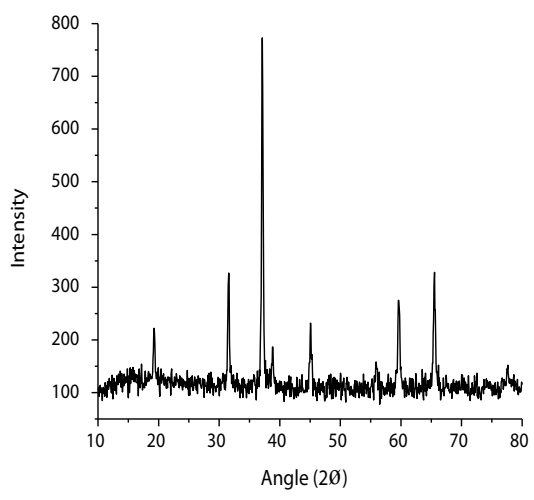

b

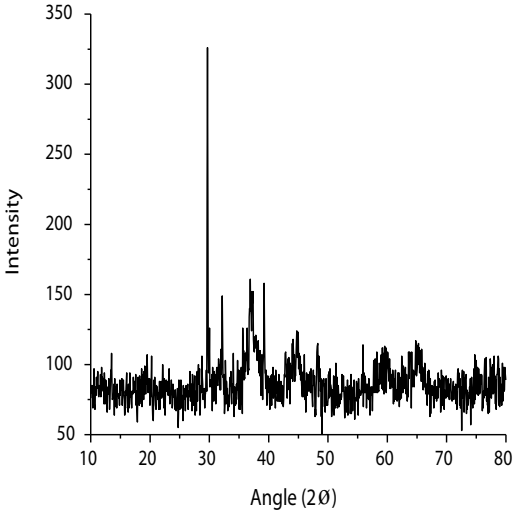

C

Figure 4: XRD spectrums of nickel oxide (a), cobalt oxide (b) and nickel-cobalt oxide (c).

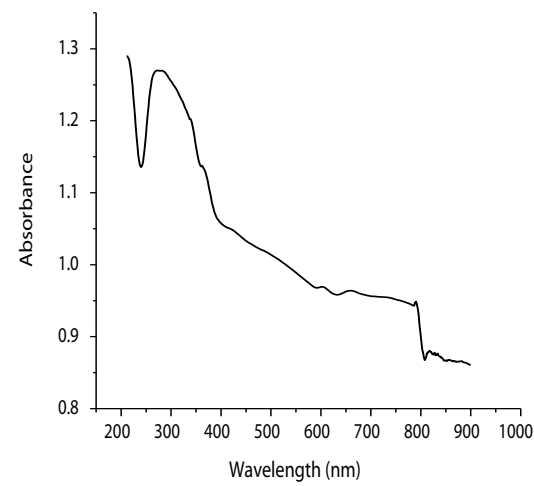

a

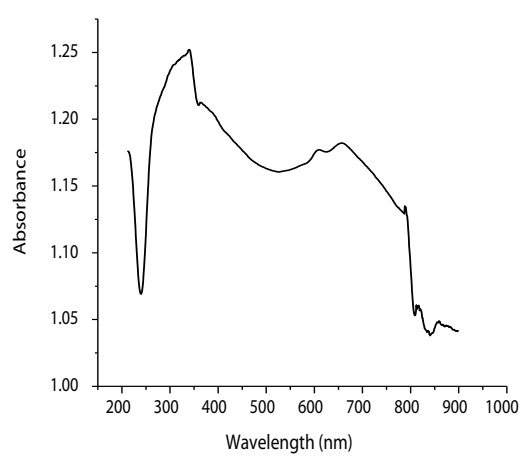

b

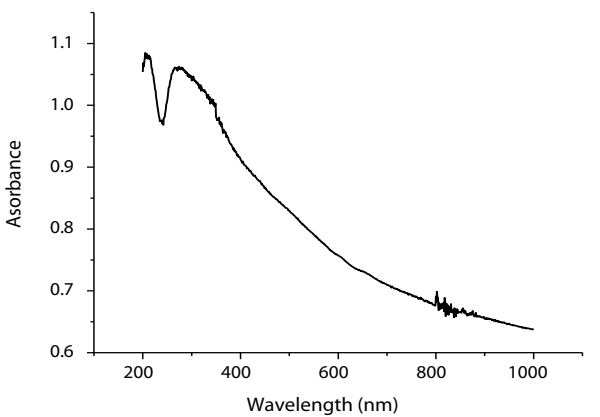

C

Figure 5: UV Spectrum of nickel oxide (a), cobalt oxide (b) and nickel-cobalt oxide (c).

oxide was $24 \mathrm{~nm}$, cobalt oxide was $20 \mathrm{~nm}$ and that of nickel - cobalt oxide was $23 \mathrm{~nm}$ respectively.

UV analysis of the oxides and nanocomposite were done. The UV spectrum of nickel oxide, cobalt oxide and the nanocomposite in the wavelength range $200 \mathrm{~nm}-900 \mathrm{~nm}$ are shown in the figure 5. The energy band of the material is related to the absorption coefficient $\alpha$ by the Tauc relation, $\alpha=A\left(h v-E_{g}\right)^{n}$, where $A$ is a constant, $h v$ is the photon energy $(v=c / \lambda), E_{g}$ is the band gap and $n$ is either 2 foran indirect transition or $1 / 2$ for a direct transition. The $(\alpha h v)^{2} v s h v$ for all the three samples is plotted and are shown in figure 6. The extrapolation of the linear portion to $h v$ axis gave value of energy gap nickel oxide as 3.89 $\mathrm{eV}$, cobalt oxide as $1.97 \mathrm{eV}$ and the nanocomposite as $1.88 \mathrm{eV}$. From the value of the band gap of the composite it could be concluded that material can be suitably used to get wide band gap materials. 


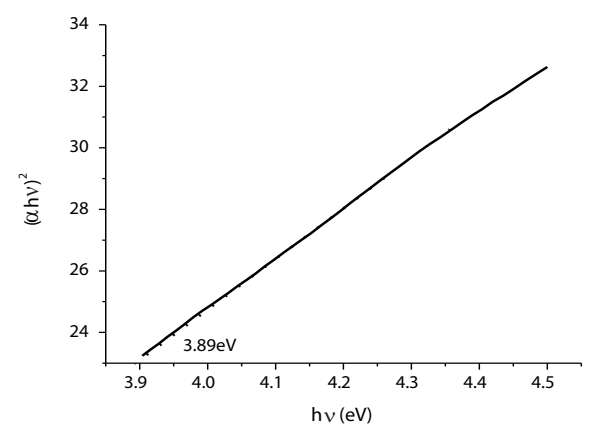

a

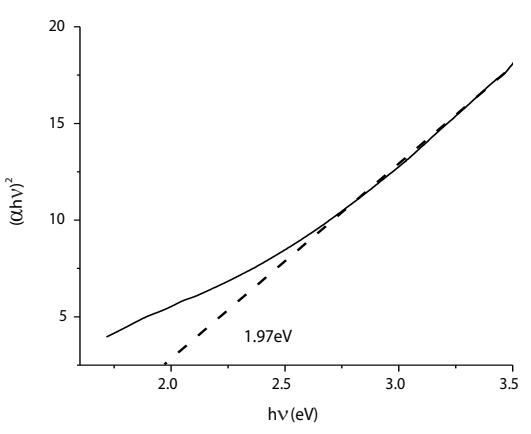

b

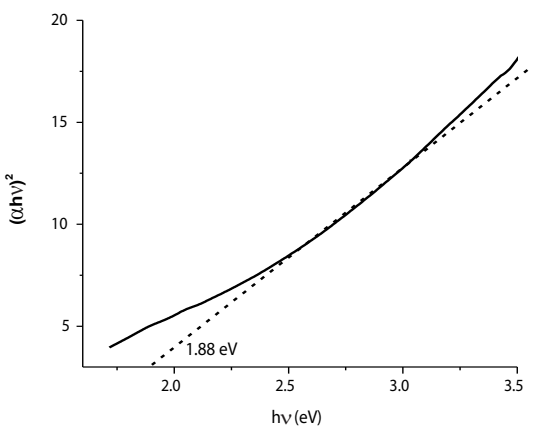

C

Figure 6: Tauc plot of nickel oxide (a), cobalt oxide (b) and nickel-cobalt oxide (c).

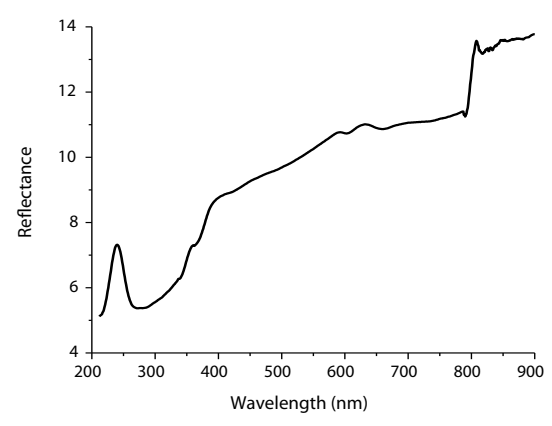

a

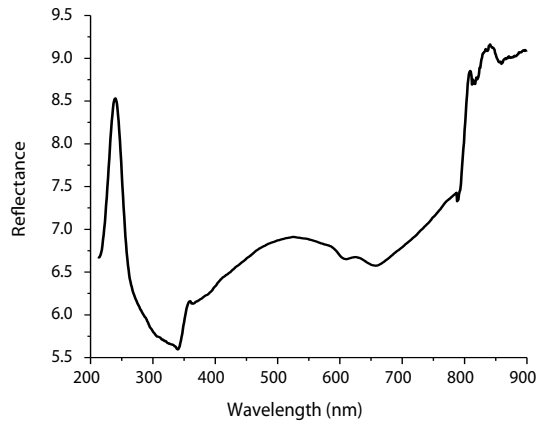

b



C

Figure 7: Reflectance Spectrum of nickel oxide (a), cobalt oxide (b) and nickel-cobalt oxide (c).

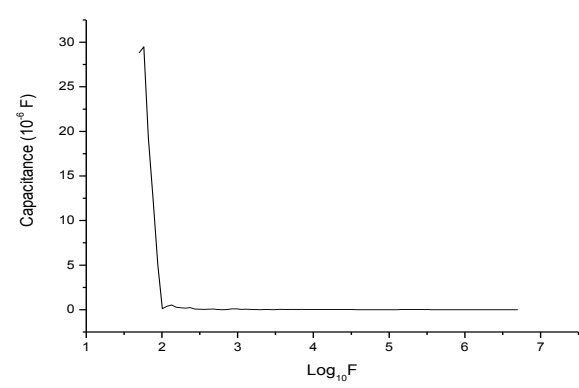

a

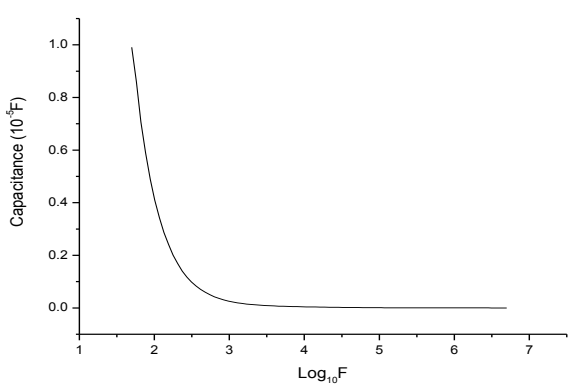

b

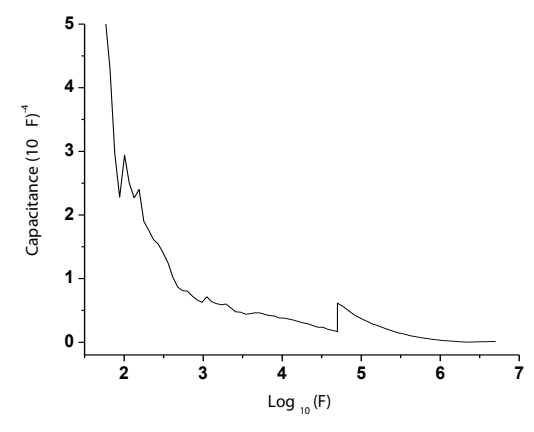

C

Figure 8: $\log _{10} \mathrm{~F}$ Vs capacitance curves of nickel oxide (a), cobalt oxide (b) and nickel-cobalt oxide (c).

The reflectance spectrum of all the three samples was also studied. The reflectance spectrums of all the three samples are shown in figure 7 respectively.

The AC conductivity studies of the samples were carried out. For this the samples annealed at $500^{\circ} \mathrm{C}$ for three hours was pelletized in presence of PVA which was then sintered at $1200^{\circ} \mathrm{C}$ for three hours and then frequency versus capacitance measurement was carried out. The results showed that the composite in as prepared form had a capacitance ranging from millifarad to picofarad in the frequency range $100 \mathrm{~Hz}$ to $13 \mathrm{MHz}$. From the result it is understood that, if properly tuned this composite can be used as a super capacitor. Conductivity also showed the corresponding variation. Figure 8 shows the frequency versus capacitance plot for annealed samples in the frequency range $100 \mathrm{~Hz}$ to $5 \mathrm{MHz}$.

\section{Conclusion}

From the crystallite size calculation using XRD spectrums, the average size of nickel oxide was $24 \mathrm{~nm}$, cobalt oxide was $20 \mathrm{~nm}$ and that of nickel-cobalt oxide was $23 \mathrm{~nm}$ respectively. From the value of the band gap of the composite it could be concluded that material can be suitably used to get wide band gap materials. From the AC conductivity study it can be concluded that the present material by proper tuning can be used as a super capacitor. Also nickel-cobalt oxide nanocomposite may be a very good magnetic nanocomposite since nickel and cobalt 
Citation: Devadatha D, Raveendran R (2013) Structural and Dielectric Characterization of Nickel-Cobalt Oxide Nanocomposite. J Material Sci Eng S11:003. doi:10.4172/2169-0022.S11-003

Page 5 of 5

in their individual state shows magnetic properties. Further studies on these materials and similar nanocomposites are needed.

\section{References}

1. Lee J, Mahendra S, Alvarez PJ (2010) Nanomaterials in the construction industry: a review of their applications and environmental health and safety considerations. ACS Nano 4: 3580-3590.

2. Karimi MA, Kargar H, Roozbahani SH, Asadiniya R, Hatefi-Mehrjardi A, et al. (2011) Synthesis and Characterization of Nanoparticles and Nanocomposite of $\mathrm{ZnO}$ and $\mathrm{MgO}$ by Sonochemical Method and their Application for Zinc Polycarboxylate Dental Cement Preparation. Int Nano Lett 1: 43-51.
3. Neamtu J, Verga N (2011) Magnetic Nanoparticles for Magneto-Resonance Imaging and Targeted Drug Delivery. Digest Journal of Nanomaterials and Biostructures 6: 969-978.

4. Jayalakshmi M, Balasubramanian K (2008) Simple Capacitors to Supercapacitors - An Overview. Int J Electrochem Sci 3: 1196-1217.

5. Fu G, Hu Z, Xie L, Jin X, Xie Y, et al. (2009) Electrodeposition of Nicke Hydroxide Films on Nickel Foil and Its Electrochemical Performances for Supercapacitor. Int J Electrochem Sci 4: 1052-1062.

6. Tang CW, Wang CB, Chien SH (2008) Characterization of cobalt oxides studied by FT-IR, Raman, TPR and TG-MS. Thermochim Acta 473: 68-73. 